\title{
Suppression of KCNQ/M (Kv7) potassium channels in the spinal cord contributes to the sensitization of dorsal horn WDR neurons and pain hypersensitivity in a rat model of bone cancer pain
}

\author{
JIE CAI ${ }^{1,2}$, DONG FANG ${ }^{1}$, XIAO-DAN LIU ${ }^{1}$, SONG LI $^{1}$, JUAN REN $^{4}$ and GUO-GANG XING ${ }^{1-3}$ \\ ${ }^{1}$ Neuroscience Research Institute, Peking University; ${ }^{2}$ Department of Neurobiology, School of Basic Medical Sciences, \\ Peking University Health Science Center; ${ }^{3}$ Key Laboratory for Neuroscience, Ministry of Education and Ministry of \\ Health, Beijing 100191; ${ }^{4}$ Cancer Center, First Hospital of Xi'an Jiaotong University, Xi'an, Shaanxi 710061, P.R. China
}

Received November 17, 2014; Accepted December 19, 2014

DOI: $10.3892 /$ or.2015.3718

\begin{abstract}
Primary and metastatic cancers that affect bones are frequently associated with severe and intractable pain. The mechanisms underlying the development of bone cancer pain are largely unknown. In the present study, we investigated whether inhibition of $\mathrm{KCNQ} / \mathrm{M}(\mathrm{Kv} 7)$ potassium channels in the spinal cord contributes to the development of bone cancer pain via sensitization of dorsal horn wide dynamic range (WDR) neurons. Using a rat model of bone cancer pain based on intratibial injection of MRMT-1 tumor cells, we observed a significant increase in C-fiber responses of dorsal horn WDR neurons in the MRMT-1 injected rats, indicating sensitization of spinal WDR neurons in bone cancer rats. Furthermore, we discovered that blockade of $\mathrm{KCNQ} / \mathrm{M}$ channels in the spinal cord by local administration of XE-991, a specific KCNQ/M channel blocker, caused a robust increase in excitability of dorsal horn WDR neurons, while, producing obvious pain hypersensitivity in normal rats. On the contrary, activation of spinal KCNQ/M channels by retigabine, a selective KCNQ/M channel opener, not only inhibited the bone cancer-induced hyperexcitability of dorsal horn WDR neurons, but also alleviated mechanical allodynia and thermal hyperalgesia in the bone cancer rats, while all of these effects of retigabine could be blocked by KCNQ/M-channel antagonist XE-991. All things considered, these results suggest that suppression of $\mathrm{KCNQ} / \mathrm{M}$ channels in the spinal cord likely contributes to
\end{abstract}

Correspondence to: Dr Guo-Gang Xing, Neuroscience Research Institute, Peking University, 38 Xue-Yuan Road, Beijing 100191, P.R. China

E-mail: ggxing@bjmu.edu.cn

Dr Juan Ren, Cancer Center, First Hospital of Xi'an Jiaotong University, Xi'an, Shaanxi 710061, P.R. China

E-mail: renjuan88@yahoo.com.cn

Key words: bone cancer pain, KCNQ/M (Kv7) potassium channel, wide dynamic range neuron, spinal cord dorsal horn, XE-991, retigabine, hyperexcitability the development of bone cancer pain via sensitization of dorsal horn WDR neurons in rats following tumor cell inoculation.

\section{Introduction}

Bone cancer pain resulting from primary tumors or tumors that metastasize to bones is one of the most severe and intractable types of cancer pain, which reduces the quality of life of patients $(1,2)$. Bone cancer pain generally consists of ongoing pain and breakthrough or incident pain (3-5), which is characterized by pathological symptoms, such as spontaneous pain, hyperalgesia and allodynia $(6,7)$. The mechanism underlying the development of bone cancer pain still remains largely unknown.

Recently, it has been observed that the thermal hyperalgesia and mechanical hypersensitivity in murine models of bone cancer pain are associated with increased excitability of primary afferent neurons that innervate the tumor-bearing limb (8-10), and reduction of KCNQ/M (Kv7) potassium channels in dorsal root ganglion (DRG) neurons leads to the hyperexcitability of primary sensory neurons, which subsequently causes sensitization of nociceptors and tumor-induced hyperalgesia under cancer conditions (11), implying that suppression of $\mathrm{KCNQ} / \mathrm{M}(\mathrm{Kv} 7)$ channels in primary DRG neurons plays a crucial role in the development of peripheral sensitization and cancer-induced bone pain.

The KCNQ/M (Kv7) channels are a family of five voltage-gated potassium $\left(\mathrm{K}^{+}\right)$channel subunits $(\mathrm{Kv} 7.1$ to Kv7.5) encoded by the KCNQ1-5 genes (12); among those, $\mathrm{KCNQ} 2$ and KCNQ3 are expressed exclusively in the nervous system $(13,14)$ and co-assembled KCNQ2 and KCNQ3 subunits are thought to constitute the native $\mathrm{M}$ channel in most neurons (15). This channel generates a species of low-threshold, slowly activating, slowly deactivating and non-inactivating $\mathrm{K}^{+}$current (the $\mathrm{M}$ current), which acts as a 'brake' to regulate the action potential (AP) firing and the neuronal excitability (16). Consequently, suppression of $M$ currents increases the neuronal excitability (16-18), whereas their enhancement has a silencing effect (19-22). In support of this notion, acute inhibition of KCNQ/M channels in primary sensory DRG neurons causes depolarization of the neuronal 
resting membrane potential and increased excitability and ultimately produces inflammatory nociception in rats $(23,24)$. Transcriptional repression of the $\mathrm{M}$ channel subunit Kv7.2 in DRG neurons contributes to the neuropathic overexcitability of peripheral sensory fibers and the maintenance of neuropathic pain in rats suffering from chronic nerve injury (25).

Apart from peripheral sensitization, central sensitization is also responsible for the generation of pain hypersensitivity and the maintenance of persistent pain $(26,27)$. In fact, sensitization of superficial dorsal horn neurons (28) and dorsal horn wide dynamic range (WDR) neurons in the spinal cord to mechanical, heat and cold stimuli have been observed in murine animals with tumor-evoked hyperalgesia $(29,30)$. On the other hand, functional $\mathrm{KCNQ} / \mathrm{M}$ channels have been detected in the dorsal horn of the spinal cord (31-35) and the central terminals of primary afferents $(36,37)$. In an in vitro study using hemisected spinal cord from immature rats, Rivera-Arconada and LopezGarcia (35) reported that blockade of KCNQ/M channels with XE-991 induced depolarization and enhanced excitability of dorsal horn neurons, whereas activation of $\mathrm{KCNQ} / \mathrm{M}$ channels with retigabine produced hyperpolarization and a large decrease in the excitability of these neurons. In the present study, we investigated whether inhibition of KCNQ/M channels in the spinal cord contributes to the development of bone cancer pain via sensitization of the dorsal horn WDR neurons. The results indicate that blockade of $\mathrm{KCNQ} / \mathrm{M}$ channels in the spinal cord produces hyperexcitability of dorsal horn WDR neurons and pain hypersensitivity in normal rats, whereas activation of these channels inhibits the sensitization of dorsal horn WDR neurons, while alleviating mechanical allodynia and thermal hyperalgesia in bone cancer rats. Thus, suppression of $\mathrm{KCNQ} / \mathrm{M}$ channels in the spinal cord leads to the sensitization of dorsal horn WDR neurons, thereby playing an important role in the development of cancer-induced bone pain.

\section{Materials and methods}

Animals. Adult female Sprague-Dawley rats weighing 180 to $220 \mathrm{~g}$ at the beginning of the experiment were provided by the Department of Experimental Animal Sciences, Peking University Health Science Center. The rats were housed in separated cages with free access to food and water. The room temperature was kept at $24 \pm 1^{\circ} \mathrm{C}$ under a natural light-dark cycle. All animal experimental procedures were conducted in compliance with the guidelines of the International Association for the Study of Pain (38) and were approved by the Animal Care and Use Committee of Peking University.

Inoculation of tumor cells. A rat model of bone cancer pain was established by intratibial injections of syngeneic MRMT-1 rat mammary gland carcinoma cells as previously described (39). Briefly, after the rat was anesthetized with chloral hydrate $(0.3 \mathrm{~g} / \mathrm{kg}$, intraperitoneally, i.p.), the left tibia was carefully exposed and a 23-gauge needle was inserted into the intramedullary canal of the bone. It was then removed and replaced with a long, thin blunt needle attached to a $10 \mu$ l Hamilton syringe containing the medium to be injected. A volume of $4 \mu \mathrm{l}$ MRMT-1 rat mammary gland tumor cells $\left(4 \times 10^{4}\right)$ or vehicle phosphorylated buffer solution (PBS) was injected into the tibial bone cavity. After injection, the site was sealed with bone wax and the wound was closed. None of the animals showed signs of motor dysfunction after inoculation of the tumor cells.

Assessment of mechanical allodynia. Mechanical allodynia, as a behavioral sign of bone cancer pain, was assessed by measuring 50\% paw withdrawal threshold (PWT) as described in our previous studies $(11,40)$. The $50 \%$ PWT in response to a series of von Frey filaments (Stoelting, Wood Dale, IL, USA) was determined by the Up and Down method (41). The rat was placed on a metal mesh floor covered with an inverted clear plastic cage $(18 \times 8 \times 8 \mathrm{~cm})$ and allowed a 20 -min period for habituation. Eight von Frey filaments with approximately equal logarithmic incremental $(0.224)$ bending forces were chosen $(0.41,0.70,1.20,2.00,3.63,5.50,8.50$ and $15.10 \mathrm{~g})$. Each trial started with a von Frey force of $2.00 \mathrm{~g}$ delivered perpendicularly to the plantar surface of the left hind paw for 2 to $3 \mathrm{sec}$. An abrupt withdrawal of the foot during stimulation or immediately after the removal of the hair was recorded as a positive response. Whenever there was a positive or negative response, the next weaker or stronger filament was applied, respectively. This procedure was carried out until 6 stimuli after the first change in response had been observed. The 50\% PWT was calculated using the following formula: $50 \% \mathrm{PWT}=10^{\left[\mathrm{X}_{\mathrm{f}}+\mathrm{k} \delta\right]}$, where $X_{f}$ is the value of the final von Frey filament used (in $\log$ units), $\mathrm{k}$ is a value measured from the pattern of positive/negative responses, and $\delta=0.224$, which is the average interval (in log units) between the von Frey filaments (42). If an animal responded to the lowest von Frey filament, a value of $0.25 \mathrm{~g}$ was assigned. If an animal did not respond to the highest von Frey filament, the value was recorded as $15.0 \mathrm{~g}$. In rats, mechanical allodynia is assessed by measuring the $50 \%$ PWT to von Frey filaments and an allodynic rat is defined as displaying a $50 \% \mathrm{PWT}<4.0 \mathrm{~g}$ (i.e., withdrawal in response to non-noxious tactile stimulus) (43).

Assessment of thermal hyperalgesia. Thermal hyperalgesia of the hind paws was tested as described in our previous study (11). Rats were allowed to acclimate for a minimum of 30 min within acrylic enclosures on a clear glass plate maintained at $30^{\circ} \mathrm{C}$. A radiant heat source was focused onto the plantar surface of the hind paw. Measurements of paw withdrawal latency (PWL) were taken by a timer that was started by the activation of the heat source and stopped when withdrawal of the paw was detected with a photodetector. A maximal cutoff time of $30 \mathrm{sec}$ was used to prevent unnecessary tissue damage. Three measurements of PWL were taken for each hind paw and were averaged as the result of each test session. The hind paw was tested alternately with $>5$ min intervals between consecutive tests.

Implantation of intrathecal catheter. Under chloral hydrate (0.3 g/kg, i.p.) anesthesia, implantation of an intrathecal cannula was performed as described in our previous study (44). Briefly, a PE-10 polyethylene catheter was implanted between lumbar (L5) and lumbar (L6) vertebrae to reach the lumbar enlargement of the spinal cord. The outer part of the catheter was plugged and fixed onto the skin on closure of the wound. All surgical procedures were performed under sterile conditions. Rats showing neurological deficits after the catheter 
implantation were euthanized. Drugs or vehicle were intrathecally injected via the implanted catheter in a 20- $\mu$ l volume of solution followed by $10 \mu \mathrm{l}$ of normal saline (NS) for flushing. Each injection lasted at least $5 \mathrm{~min}$. After the injection, the needle remained in situ for 2 min before being withdrawn.

Measurement of drug effects on pain behaviors. The first behavioral experiment was performed to examine whether inhibition of $\mathrm{KCNQ} / \mathrm{M}$ channels in the spinal cord with a potent blocker XE-991 would produce pain hypersensitivity in normal rats. XE-991 (at $0.9 \mu \mathrm{g} / \mu \mathrm{l}$ ) was intrathecally administered to the animal on day 7 after intrathecal cannula implantation and the effects of XE-991 on ipsilateral PWT and PWL of the rats were measured at $0.5,1.5,2.5$, and $3.5 \mathrm{~h}$ after drug injection, respectively. The second experiment was carried out to determine whether activation of spinal $\mathrm{KCNQ} / \mathrm{M}$ channels by using its specific opener retigabine (RTG) would attenuate the hyperalgesic behaviors in a rat model of bone cancer pain, and whether the effects of retigabine could be reversed by the KCNQ/Mchannel blocker XE-991. On day 14 after tumor cell inoculation, rats exhibiting bone cancer pain, confirmed by measuring the mechanical allodynia and thermal hyperalgesia, were intrathecally administered with RTG, RTG plus XE-991, or vehicle DMSO. Here, RTG was used at a concentration of $8.5 \mu \mathrm{g} / \mu 1$ and XE-991 at $0.9 \mu \mathrm{g} / \mu \mathrm{l}$, and the effects of RTG on ipsilateral PWT and PWL of rat were measured at $0.5,1.5,2.5$ and $3.5 \mathrm{~h}$ after drug injection, respectively. To examine the long-term effects of intrathecal RTG on bone cancer-induced pain hypersensitivity in the MRMT-1-inoculated rats, RTG (at $8.5 \mu \mathrm{g} / \mu \mathrm{l}$ ) was intrathecally administered to the rats twice per day at a 30-min interval, lasted for 3 days, and the effect of RTG on both PWT and PWL was tested on day 4 after drug injection.

Assessment of locomotor function. Inclined-plate test was used for the assessment of locomotor function. Rat was placed crosswise to the long axis of an inclined plate. The initial angle of the inclined plate was 50 degrees. The angle was then adjusted in 5-degree increments. The maximum angle of the plate on which the rat maintained its body position for $5 \mathrm{sec}$ without falling was determined according to the method reported by Rivlin and Tator (45). In this study, inclined-plate test was performed for all behavioral experiments in which drugs were intrathecally administrated to rats.

\section{Electrophysiological studies}

Surgery. Each rat was initially anesthetized with urethane (1.2-1.5 g/kg, i.p.). The trachea was cannulated to allow mechanical ventilation with room air. A catheter was inserted into the right jugular vein for continuous infusion of Tyrode's solution [in mmol/l: $\mathrm{NaCl} 137, \mathrm{KCl} 2.7, \mathrm{CaCl}_{2} 1.4, \mathrm{MgCl}_{2} 1.0$, $\mathrm{NaHCO}_{3}$ 6.0, $\mathrm{NaH}_{2} \mathrm{PO}_{4}$ 2.1, D-(+)-glucose 6.5] with a pH of 7.4 at $1.0-1.5 \mathrm{ml} / \mathrm{h}$. The rectal temperature was maintained at $36.5-37.5^{\circ} \mathrm{C}$ via a feedback controlled under a body heating pad. A pair of bipolar silver hook electrodes was placed under sciatic nerve immediately proximal to the trifurcation for the electrical stimulation. The vertebral column was rigidly fixed in the frame with two clamps. The lumbar enlargement of the spinal cord was exposed by laminectomy at vertebrae $\mathrm{T} 13$ and L1 and the dura covering the lumbosacral spinal segments was carefully removed. A small well was built with $3 \%$ agar on the dorsal spinal cord at the recording segment to allow application of drugs or vehicles $(44,46)$. The exposed spinal tissue was covered with warm $\left(37^{\circ} \mathrm{C}\right)$ saline solution. After surgery, the animal was artificially ventilated with a small animal ventilator and continuous anesthesia was maintained with urethane $(0.10-0.15 \mathrm{~g} / \mathrm{kg} / \mathrm{h})$ during the whole experiment. The depth of anesthesia was monitored by examination of pupillary size and reflexes. The physiological condition of the animal was monitored by recording the electrocardiogram (330-460 beats/min), end-expiratory $\mathrm{CO}_{2}$ (3.5-4.5\%), and rectal temperature $\left(36.5-37.5^{\circ} \mathrm{C}\right)$ and was maintained within the range indicated (44).

Extracellular recording. Single-unit extracellular recordings were conducted from the lumbar dorsal horn neurons within $1,200 \mu \mathrm{m}$ of the dorsal surface of the spinal cord with 2-5 M parylene-coated tungsten microelectrode (Friedrick Haer \& Co. Inc., Bowdoinham, ME, USA). The microelectrode was inserted perpendicularly into the dorsal horn from a point about midway between the midline and the medial edge of the dorsal root entry zone. During electrode advancement, electrical pulses $(0.5 \mathrm{~Hz}, 0.3 \mathrm{msec}$ pulse width, $0.4 \mathrm{~mA})$ were applied to the ipsilateral sciatic nerve as search stimuli so that a neuron with no spontaneous firing could be identified. Once a single unit was identified, the receptive field and response characteristics were determined by a range of mechanical stimuli of various intensities, including brushing or touching the skin with a cotton brush, light pressure with a probe and pinching a fold of skin with toothed forceps. A neuron responding to innocuous tactile stimuli, light pressure and noxious pinch in a graded manner was identified as a WDR neuron and was selected for further investigation (44). The recorded signals were first amplified with an AC pre-amplifier and then filtered with a band pass filter at 500-1,000 Hz. The filtered signals were displayed on an oscilloscope and fed to a Pentium computer via a CED 1401 interface for off-line analysis using the Spike2 software (Cambridge Electronic Design, Cambridge, UK). Spikes appearing 45-300 msec after stimulus were defined as $\mathrm{C}$-fiber responses, i.e. responses in the WDR neurons evoked by C-fiber activation (44). Single cell recording was confirmed on the basis of amplitude and shape of the action potentials. In the following electrophysiological studies, only one cell was studied in each animal and each animal received only one dose of RTG, XE-991 or vehicle.

Measurement of drug effects. The electrophysiological experiments were designed to investigate the effects of spinal administration of XE-991 and RTG on the activities of WDR neurons in naive rats and in rats with bone cancer pain, respectively. In this experiment, a train of 10 stimuli $(0.5 \mathrm{~Hz}$, 0.5 -msec pulse width, with a pulse current of $2 \mathrm{x}$ C-fiber response threshold), which was used as the test stimulus, was applied repeatedly to the sciatic nerve at a 5-min interval, and post-stimulus histograms from the responses of WDR neurons were generated by the Spike2 software. After six stable control responses were recorded, XE-991 at the dose of $0.4 \mathrm{mM}$ (33) or RTG at the dose of $5 \mathrm{mM}$ (32) in a $50 \mu \mathrm{l}$ volume of solution, or the equal volume of vehicle, was applied topically to the dorsal surface of the spinal cord. The post-drug responses evoked by the same test stimulus as described above were measured at 
$5 \mathrm{~min}$ intervals for up to $60 \mathrm{~min}$. In the present study, only C-fiber responses of WDR neurons, which are highly related to nociceptive transmission $(44,47)$ were examined and analyzed. All of the C-fiber responses were expressed as percentages of the mean response value of six pre-drug consecutive trains of test stimuli.

Chemical preparation and application. Retigabine (N-(2-amino-4-(4-fluorobenzylamino)-phenyl)carbamic acid ethyl ester, Melone Phamaceutical Co., Ltd., Dalian, China) and XE-991 (10,10-bis(4-pyridinyl-methyl)-9(10H)-anthracenone, Tocris, Ellisville, MO, USA) were dissolved in DMSO(dimethyl sulfoxide; Sigma Co.) as a $0.5 \mathrm{M}$ stock solution, stored at $-20^{\circ} \mathrm{C}$ and diluted to the desired concentrations with normal saline just before the experiments. The final concentration of DMSO was $<0.5 \%$. All other chemicals or reagents were obtained from Sigma, Invitrogen or Abcam except as mentioned in the text.

Statistical analysis. Statistical analyses were performed with GraphPad Prism 5 for Windows (GraphPad Software, La Jolla, CA, USA). All data are expressed as mean \pm SEM. The two-tailed Student t-test was used for the comparison of the mean values between the two groups. One-way analysis of variance (ANOVA) followed by the Dunnett multiple comparison test or two-way ANOVA followed by Bonferroni post-hoc test was used for multiple comparisons. Differences with $\mathrm{P}<0.05$ were considered statistically significant. The area under the time-course curve (AUC) during the analysis time was used for assessing the summed effects of XE-991 and retigabine as previously described (44).

\section{Results}

Increase in excitability of dorsal horn WDR neurons in the spinal cord in bone cancer rats. First, we examined the $\mathrm{C}$-fiber responses of dorsal horn WDR neurons, which are highly related to nociceptive transmission $(44,47)$ in rats with cancer-induced bone pain. We found that the $\mathrm{C}$-fiber responses of WDR neurons were significantly increased in the MRMT-1 tumor cell inoculated rats compared to the naive and PBS-treated rats (Fig. 1). As shown in Fig. 1D, the total spike count of the electrically evoked C-fiber responses in 10 stimuli was significantly increased in the MRMT-1 rats compared to the naive and PBS-treated rats $(\mathrm{P}<0.001$, two-way ANOVA). As summarized in the AUC of $\mathrm{C}$-fiber responses, the AUC (0-55 min of the analysis time) of the $\mathrm{C}$-fiber responses in the MRMT-1-inoculated rats was also increased prominently $(11,700 \pm 590.7)$ compared to the naive $(3,343 \pm 463.7)$ and the PBS-treated rats $(3,991 \pm 318.2)(\mathrm{P}<0.001$, one-way ANOVA; Fig. 1E). Representative examples illustrating the C-fiber responses of dorsal horn WDR neurons in the naive, PBS- and MRMT-1-inoculated rats are shown in Fig. 2A-C respectively. These results suggest that hyperexcitability of dorsal horn WDR neurons emerges in the tumor cell inoculated rats, which likely underlies the development of central sensitization and cancer-induced bone pain.

Blockade of spinal KCNQ/M channels with XE-991 induces hyperexcitability of dorsal horn WDR neurons in normal rats.
Next, we investigated whether repressed $\mathrm{KCNQ} / \mathrm{M}$ channels in the spinal cord contribute to the hyperexcitability of dorsal horn WDR neurons. We found that spinal administration of XE-991 (at $0.4 \mathrm{mM}$ ), a potent $\mathrm{KCNQ} / \mathrm{M}$ channel blocker, induced a significant increase in C-fiber responses of dorsal horn WDR neurons in the normal rats (Fig. 2). The enhanced effect of XE-991 on C-fiber responses of WDR neurons began at $10 \mathrm{~min}$ post-XE-991 (131.09 $\pm 6.2 \%$ of baseline), maintained for $60 \mathrm{~min}(177.74 \pm 11.5 \%$ of baseline) until termination of the experiment. On the contrary, spinal administration of vehicle DMSO had no significant effect on the C-fiber responses of WDR neurons ( $P<0.05-0.001$, two-way ANOVA; Fig. 2E). As summarized in the AUC of the C-fiber responses, the AUC (-25-60 min of the analysis time) was also increased markedly in the XE-991 group $(11,970 \pm 521.4)$ compared to the DMSO group $(8,689 \pm 223.2) \quad(\mathrm{P}<0.001, \mathrm{XE}-991$ vs. DMSO, two-tailed unpaired t-test; Fig. 2F). Representative examples illustrating the $\mathrm{C}$-fiber responses of dorsal horn WDR neurons before and after administration of XE-991 or DMSO are shown in Fig. 2A-D. These results imply that repression of spinal KCNQ/M channels may induce enhanced excitability of dorsal horn WDR neurons in normal rats.

Intrathecal administration of XE-991 produces mechanical allodynia and thermal hyperalgesia in normal rats. Given that blockade of spinal KCNQ/M channels with XE-991 induces hyperexcitability of dorsal horn WDR neurons, we hypothesized that intrathecal administration of XE-991 would produce pain hypersensitivity in normal rats. As our expectation, spinal application of XE-991 produced a statistical decrease in ipsilateral PWT (in $\mathrm{g}$ ) from $0.5 \mathrm{~h}$ after drug injection $(4.45 \pm 0.65$ XE-991 vs. $15.10 \pm 0.01 \mathrm{DMSO}, \mathrm{P}<0.001)$ and lasted for $3.5 \mathrm{~h}$

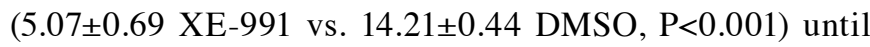
termination of the experiment (two-way ANOVA, n=11/group; Fig. 3A). As summarized in the AUC of PWT, the AUC (0-3.5 $\mathrm{h}$ of the analysis time) was significantly decreased in the XE-991 group $(17.85 \pm 1.79)$ compared to the DMSO group $(50.70 \pm 1.11)(\mathrm{P}<0.001$, two-tailed unpaired t-test; Fig. 3C). Similarly, intrathecal injection of XE-991 also produced a remarkable decrease in ipsilateral PWL (in sec) from $0.5 \mathrm{~h}$ $(11.19 \pm 0.91 \mathrm{XE}-991$ vs. $21.58 \pm 1.11 \mathrm{DMSO}, \mathrm{P}<0.001)$ to $3.5 \mathrm{~h}$ $(10.74 \pm 1.22$ XE-991 vs. $21.93 \pm 0.8 \mathrm{DMSO}, \mathrm{P}<0.001)$ following drug administration (two-way ANOVA, n=9/group; Fig. 3B). The AUC (0-3.5 h of the analysis time) of PWL was prominently decreased in the XE-991 group $(38.92 \pm 2.58)$ compared to the DMSO group $(74.35 \pm 2.79) \quad(\mathrm{P}<0.001$, two-tailed unpaired t-test, Fig. 3D). Moreover, the inclined-plate test was performed at $30 \mathrm{~min}$ before and $4 \mathrm{~h}$ after drug injection to assess the effect of XE-991 on the locomotor function of the rats. The results showed that intrathecal injection of XE-991 at our experimental dose had no obvious damage to the locomotor function of the rats $(\mathrm{P}>0.05$, two-tailed unpaired t-test, pre-drug vs. post-drug, $n=11 /$ group, Fig. $3 \mathrm{E}$ ). These data indicate that suppression of spinal KCNQ/M channels also produces mechanical allodynia and thermal hyperalgesia in normal rats.

Activation of spinal $\mathrm{KCNQ} / \mathrm{M}$ channels with retigabine reduces the bone cancer-induced hyperexcitability of dorsal horn WDR neurons in MRMT-1-inoculated rats. To further 
A

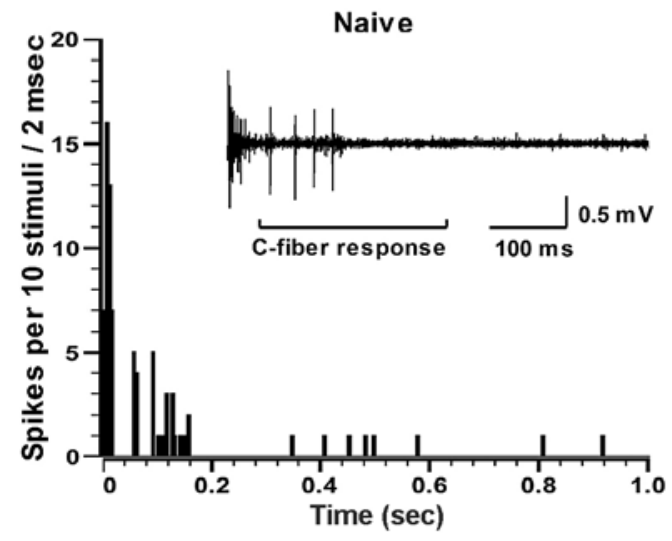

C

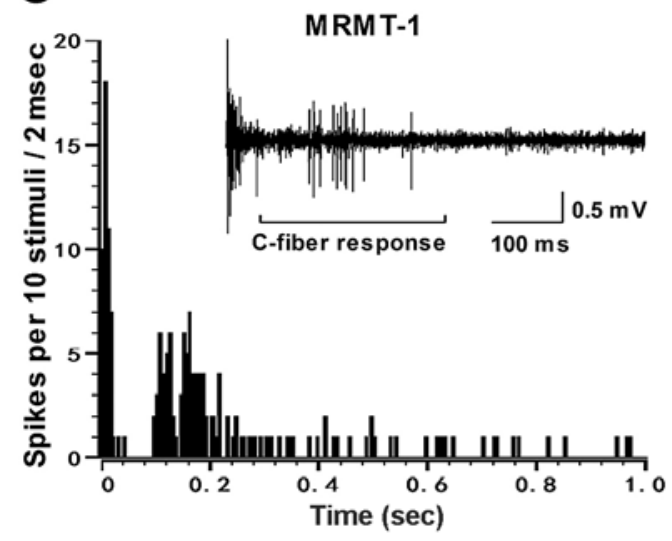

B

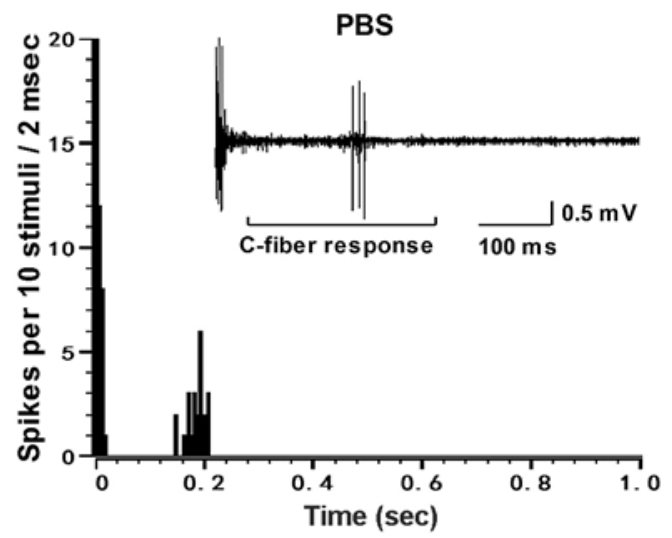

D

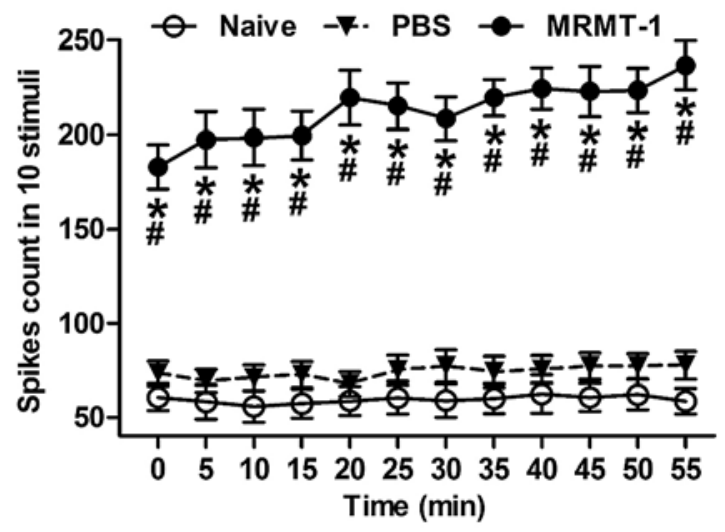

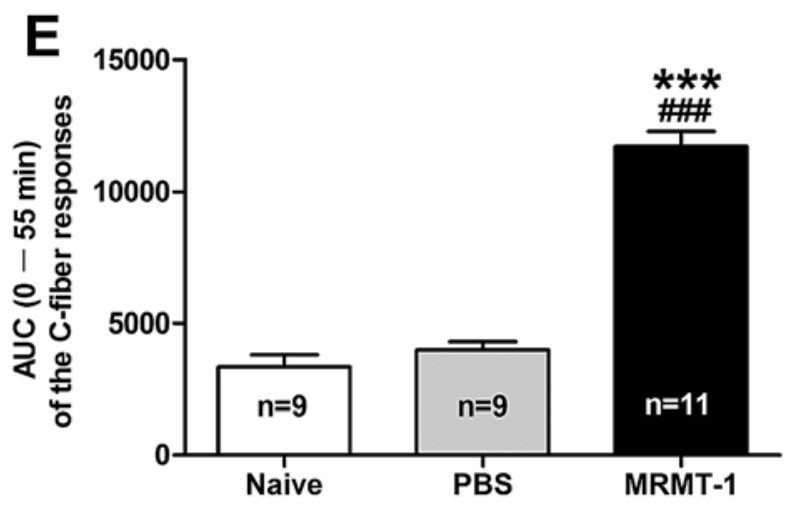

Figure 1. Alterations of the C-fiber responses of dorsal horn WDR neurons in bone cancer rats. (A-C) Representative C-fiber responses of dorsal horn WDR neurons in naive (A), PBS (B) and MRMT-1 tumor cell (C) inoculated rats. Panels illustrate the post-stimulus histogram of the electrically evoked neuronal responses. Inset shows the original recordings of the first electrically evoked neuronal responses. (D) The total spike count of the electrically evoked C-fiber responses in 10 stimuli. ${ }^{* / \#} \mathrm{P}<0.001$, compared to the naive and the PBS group, respectively, two-way ANOVA followed by Bonferroni post-hoc test, $\mathrm{n}=9-11 /$ group. (E) AUC (0-55 min) of the C-fiber responses. ${ }^{* * * \# \# \# ~} \mathrm{P}<0.001$, compared to the naive and the PBS group, respectively, one-way ANOVA followed by the Dunnett multiple comparison test, $\mathrm{n}=9-11$ /group. AUC, area under the time-course curve. WDR, wide dynamic range; PBS, phosphorylated buffer solution; ANOVA, analysis of variance.

determine whether the enhanced excitability of dorsal horn WDR neurons in rats with bone cancer pain depends on the repression of spinal $\mathrm{KCNQ} / \mathrm{M}$ channels, we tested the effects of RTG, a selective $\mathrm{KCNQ} / \mathrm{M}$ channel opener, on $\mathrm{C}$-fiber responses of WDR neurons in MRMT-1-inoculated rats. As shown in Fig. 4, spinal administration of RTG (at $5 \mathrm{mM}$ ) significantly reduced the enhanced excitability of dorsal horn WDR neurons in bone cancer rats. The inhibitory effect of RTG on C-fiber responses of WDR neurons began at $10 \mathrm{~min}$
post-RTG $(83.82 \pm 4.5 \%$ of baseline), lasted for $60 \mathrm{~min}$ $(66.23 \pm 3.2 \%$ of baseline) until termination of the experiment. In contrast, spinal administration of vehicle DMSO had no significant effect on the $\mathrm{C}$-fiber responses of WDR neurons in the MRMT-1-inoculated rats $(\mathrm{P}<0.01-0.001, \mathrm{RTG}$ vs. DMSO, two-way ANOVA; Fig. 4E). As summarized in AUC of the C-fiber responses, the AUC (-25-60 min of the analysis time) was significantly decreased in the RTG group $(6,978 \pm 148.5)$ compared to the DMSO group $(8,648 \pm 151.6)(\mathrm{P}<0.001, \mathrm{RTG}$ 

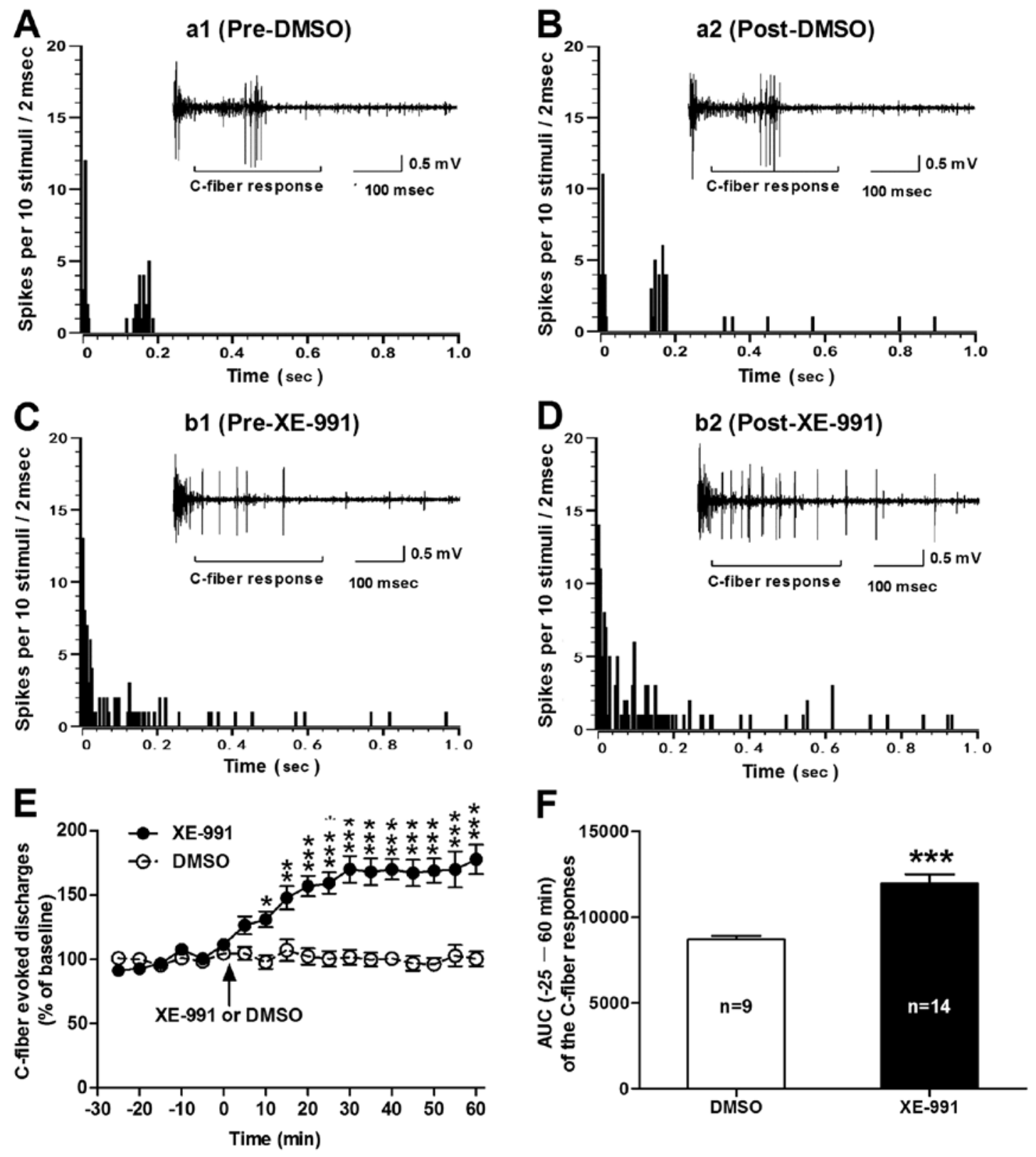

Figure 2. Effects of spinal administration of XE-991 on the C-fiber responses of dorsal horn WDR neurons in normal rats. (A-D) Post-stimulus histograms of the electrically evoked neuronal responses in a dorsal horn WDR neuron before (a1, DMSO; b1, XE-991) and after (a2, DMSO; b2, XE-991) drug application. Inset shows the original recordings of the first electrically evoked neuronal responses corresponding to each time-point respectively. (E) Analysis of the C-fiber evoked discharges before and after drug (XE-991 or DMSO) application. Note that spinal administration of XE-991 (0.4 mM) induced a significant increase in $\mathrm{C}$-fiber responses of dorsal horn WDR neurons in normal rats. ${ }^{*} \mathrm{P}<0.05,{ }^{* *} \mathrm{P}<0.01$ and ${ }^{* * * *} \mathrm{P}<0.001$, compared to control DMSO, two-way ANOVA followed by Bonferroni post-hoc test, $\mathrm{n}=9$-14/group). (F) AUC (-25-60 min) of the C-fiber responses. ${ }^{* * *} \mathrm{P}<0.001$, XE-991 vs. DMSO, two-tailed unpaired t-test, $\mathrm{n}=9 \mathrm{DMSO}$ and 14 XE-991. WDR, wide dynamic range; ANOVA, analysis of variance; AUC, area under the time-course curve.

vs. DMSO, two-tailed unpaired t-test; Fig. 4F). Representative examples illustrating the $\mathrm{C}$-fiber responses of dorsal horn WDR neurons before and after administration of RTG or DMSO in the MRMT-1-inoculated rats are shown in Fig. 4A-D. The results indicate that activation of spinal $\mathrm{KCNQ} / \mathrm{M}$ channels with RTG may inhibit the bone cancer-induced hyperexcitability of dorsal horn WDR neurons in MRMT-1-inoculated rats.

Intrathecal administration of retigabine alleviates mechanical allodynia and thermal hyperalgesia in bone cancer rats. Based on the aforementioned findings that activation of spinal $\mathrm{KCNQ} / \mathrm{M}$ channels with RTG inhibits the sensitization of dorsal horn WDR neurons in bone cancer rats, we speculated that intrathecal administration of RTG would attenuate the bone cancer-induced pain hypersensitivity in the MRMT-1-inoculated rats. As shown in Fig. 5, spinal application of RTG rescued the bone cancer-induced decrease in ipsilateral PWT and PWL in the MRMT-1-inoculated rats. The decreased PWT (in g) was restored from $0.5 \mathrm{~h}(10.39 \pm 1.13$ RTG vs. 2.92 $\pm 0.57 \mathrm{DMSO}, \mathrm{P}<0.001)$ to $2.5 \mathrm{~h}(6.32 \pm 0.59$ RTG vs. $2.99 \pm 0.80 \mathrm{DMSO}, \mathrm{P}<0.05)$ after intrathecal injection of RTG compared to vehicle DMSO (two-way ANOVA, $\mathrm{n}=9$ /group; Fig. $5 \mathrm{~A})$. The AUC (0-3.5 $\mathrm{h}$ of the analysis time) 
A

Mechanical allodynia

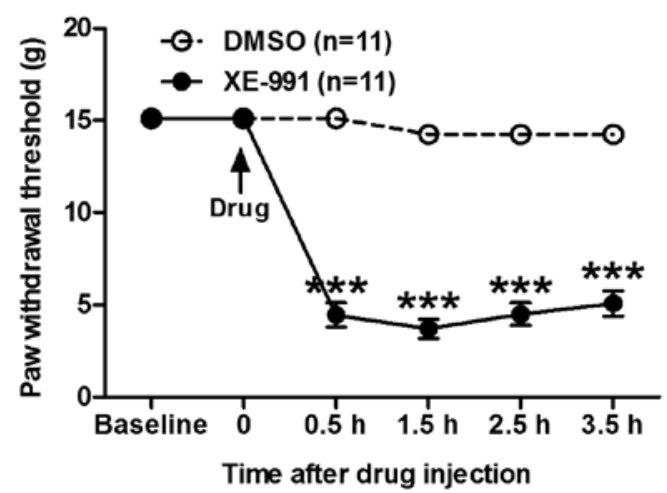

C

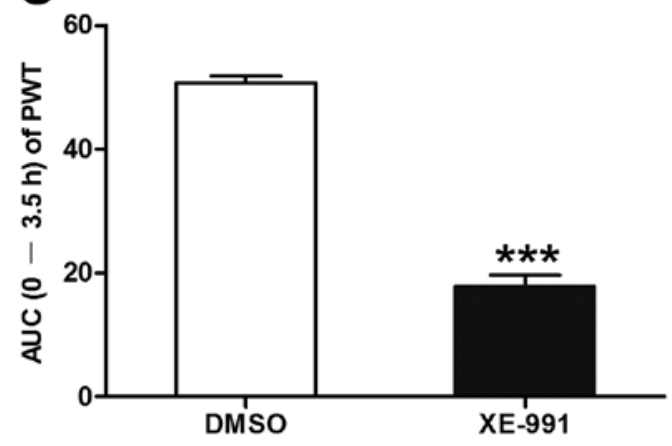

B

\section{Thermal hyperalgesia}

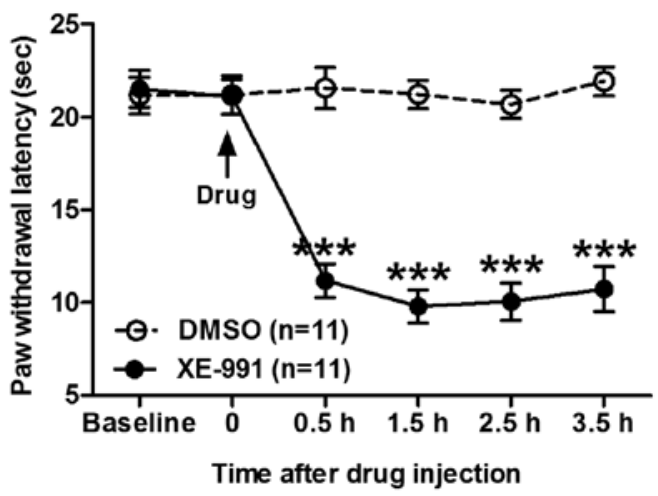

D

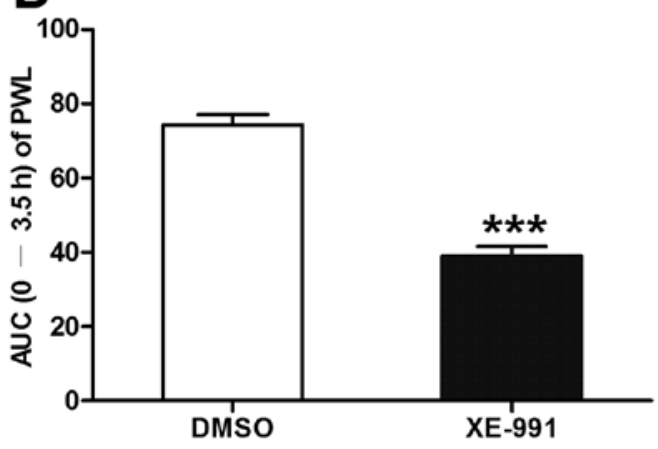

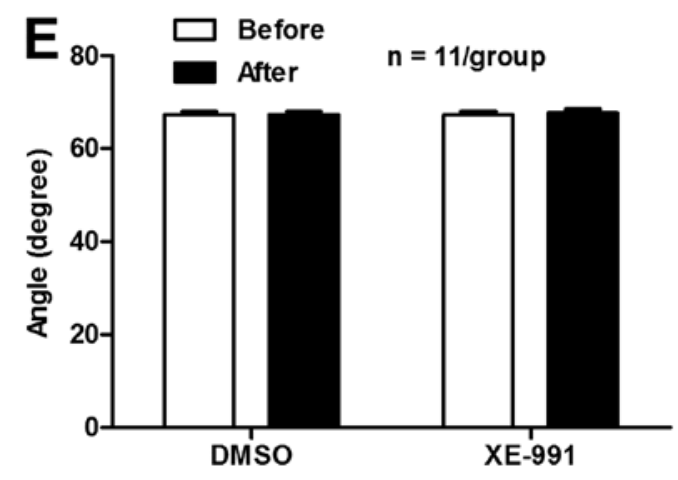

Figure 3. Effects of intrathecal administration of XE-991 on ipsilateral PWT and PWL in normal rats. (A and B) The time course of spinal application of XE-991 $(0.9 \mu \mathrm{g} / \mu \mathrm{l})$ on PWT (A) and PWL (B). Note that intrathecal administration of XE-991 produced obvious mechanical allodynia and thermal hyperalgesia in normal rats. ${ }^{* * *} \mathrm{P}<0.001$, compared to the vehicle DMSO group, two-way ANOVA followed by Bonferroni post-hoc test, $\mathrm{n}=11 / \mathrm{group}$. (C and $\left.\mathrm{D}\right)$ Area under the time-course curve (AUC) of PWT (C) and PWL in A and B, respectively. ${ }^{* * *} \mathrm{P}<0.001$, compared to the vehicle DMSO group, two-tailed unpaired $\mathrm{t}$-test, $\mathrm{n}=11 /$ group. (E) Effects of spinal XE-991 on the locomotor function of rats measured by the angle of the inclined-plate at which the animal begins to fall. Note that intrathecal injection of XE-991 had no significant damage on the locomotor function of the rats $(\mathrm{P}>0.05$, pre-drug vs. post-drug, two-tailed unpaired t-test, $\mathrm{n}=11$ /group). PWT, paw withdrawal threshold; PWL, paw withdrawal latency; ANOVA, analysis of variance; AUC, area under the time-course curve.

of PWT was significantly decreased in the RTG group $(24.47 \pm 2.46)$ compared to the DMSO group $(10.17 \pm 2.34)$ $(\mathrm{P}<0.01$, one-way ANOVA, $\mathrm{n}=9 /$ group; Fig. $5 \mathrm{C})$. Moreover, the inhibitory effect of RTG on the reduction of PWT in bone cancer rats was almost completely blocked by the KCNQ/ M-channel antagonist XE-991 (Fig. 5A and C). Similarly, RTG also inhibited the reduction of PWL in the bone cancer rats, and this inhibitory effect of RTG was also blocked by XE-991 (Fig. 5B and D). To examine the long-term effects of intrathecal RTG on bone cancer-induced pain hypersensitivity in the MRMT-1-inoculated rats, RTG at $8.5 \mu \mathrm{g} / \mu \mathrm{l}$ was intrathecally administrated to rats twice/day at a 30 -min interval, for 3 days, and the effect of RTG on both PWT and PWL was tested on day 4 after drug injection. The results showed that repeated administration of RTG for 3 days produced a longterm anti-allodynic and anti-hyperalgesic effects in the bone cancer rats. As shown in Fig. 5E and F, both the decreased PWT (7.59 \pm 0.70 vs. $3.10 \pm 0.56$ g, RTG vs. DMSO, P<0.001) and PWL $(15.40 \pm 1.26$ vs. $9.13 \pm 0.97$ s, RTG vs. DMSO, P<0.001) in the MRMT-1-treated rats were significantly restored on day 4 after repeated application of RTG compared to vehicle DMSO (two-way ANOVA, n=9/group). Similarly, XE-991 blocked the inhibitory effects of RGT on both the decreased PWT and PWL in the bone cancer rats (Fig. 5E and F), and no significant 

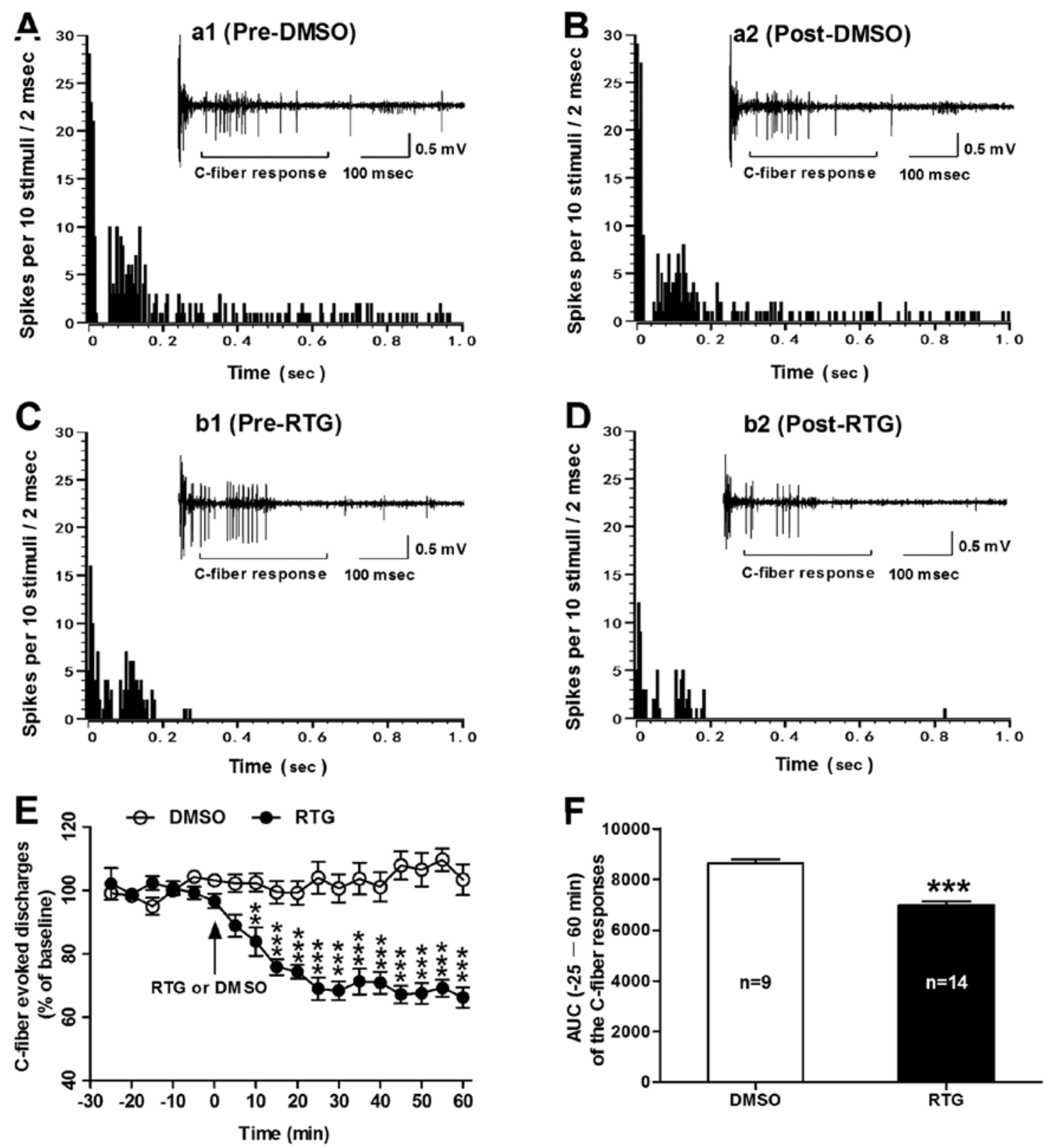

Figure 4. Effects of spinal administration of RTG on C-fiber responses of dorsal horn WDR neurons in bone cancer rats. (A-D) Post-stimulus histograms of the electrically evoked neuronal responses in a dorsal horn WDR neuron before (a1, DMSO; b1, RTG) and after (a2, DMSO; b2, RTG) drug application. Inset shows the original recordings of the first electrically evoked neuronal responses corresponding to each time point respectively. (E) Analysis of the C-fiber evoked discharges before and after drug (RTG or DMSO) application in MRMT-1 tumor cell inoculated rats. Note that spinal administration of RTG (5 mM) significantly reduced the enhanced excitability of dorsal horn WDR neurons in the bone cancer rats. ${ }^{* *} \mathrm{P}<0.01,{ }^{* * * *} \mathrm{P}<0.001$, compared to the vehicle DMSO group, two-way ANOVA followed by Bonferroni post-hoc test, $\mathrm{n}=9-14$ /group). (F) AUC (-25-60 min) of the C-fiber responses. ${ }^{* * *} \mathrm{P}<0.001$, RTG vs. DMSO, two-tailed unpaired t-test, n=9-14/group. RTG, retigabine; WDR, wide dynamic range; ANOVA, analysis of variance; AUC, area under the time-course curve.

damage was observed in the locomotor function of rats after repeated application of RTG (Fig. 5G). These results suggest that activation of the spinal KCNQ/M channels by RGT alleviates both mechanical allodynia and thermal hyperalgesia in bone cancer rats.

\section{Discussion}

As extension of our previous findings showing that inoculation of tumor cells into the tibial canal in rats induces enhanced excitability of primary sensory DRG neurons, which is responsible for the peripheral sensitization and tumor-induced hyperalgesia under cancer conditions (10), our present study provides electrophysiological evidence confirming the sensitization of dorsal horn WDR neurons (i.e., central sensitization) in MRMT-1 tumor cell inoculated rats, which is considered as another underlying mechanism for the development of cancer-induced bone pain $(7,29)$. A marked increase in the C-fiber responses of WDR neurons to electrical stimulation of the sciatic nerve in bone cancer rats was observed. This is consistent with a previous study showing that, in a mouse model of bone cancer pain established by implantation of fibrosarcoma cells into the calcaneus bone, WDR, but not high threshold (HT), nociceptive dorsal horn neurons in 

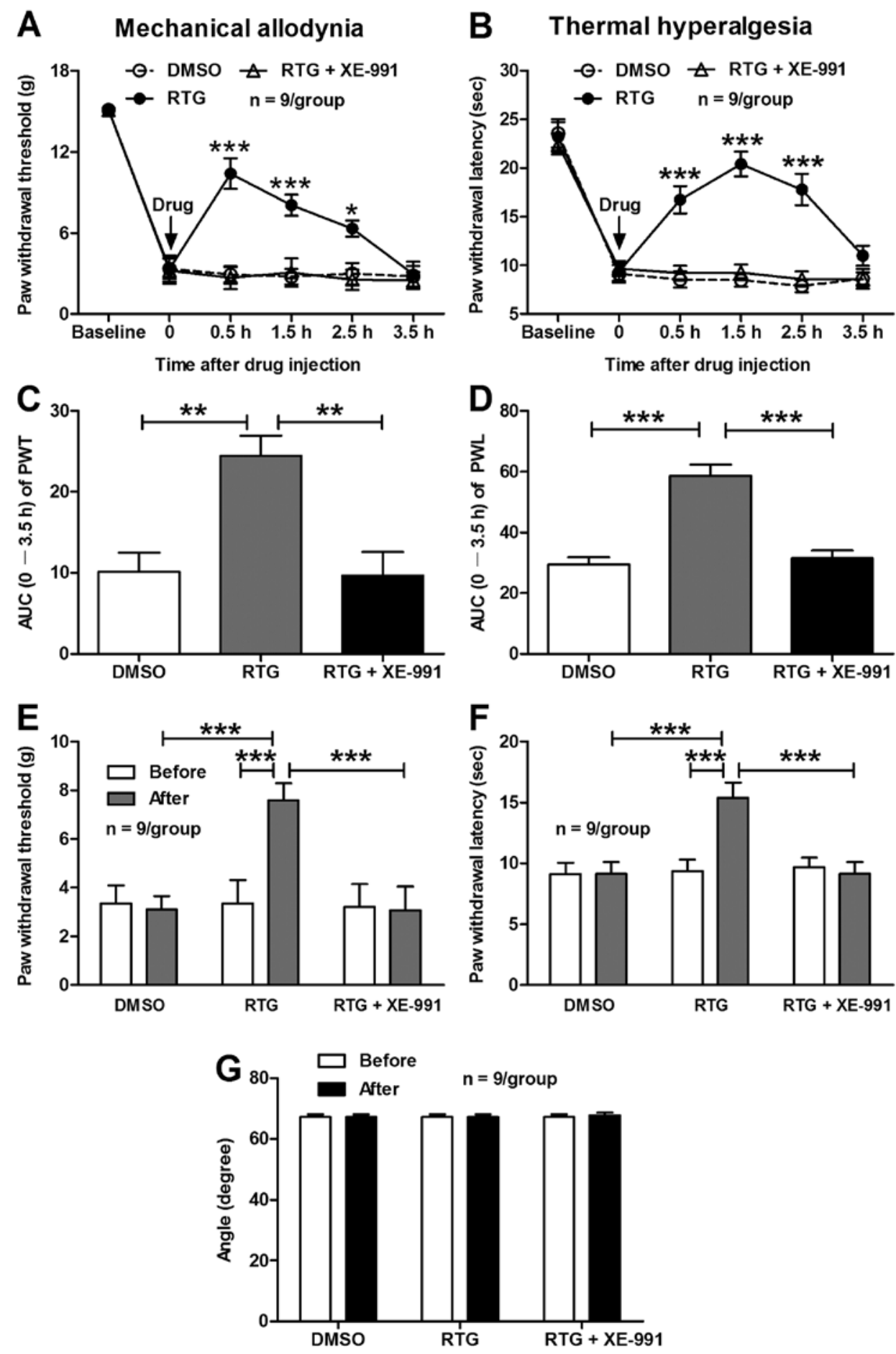

Figure 5. Effects of intrathecal administration of RTG on ipsilateral PWT and PWL in bone cancer rats. (A and B) The time course of spinal application of RTG $(8.5 \mu \mathrm{g} / \mu \mathrm{l})$ on PWT (A) and PWL (B). Note that RTG significantly inhibited the bone cancer-induced reduction of ipsilateral PWT (A) and PWL (B) in MRMT-1-inoculated rats and all the effects of RTG were blocked by XE-991. ${ }^{*} \mathrm{P}<0.05$ and ${ }^{* * *} \mathrm{P}<0.001$, compared to the vehicle DMSO group, two-way ANOVA followed by Bonferroni post-hoc test, $\mathrm{n}=9$ /group. (C and D) Area under the time-course curve (AUC) of PWT and PWL in A and B, respectively. ${ }^{* *} \mathrm{P}<0.01$ and ${ }^{* * *} \mathrm{P}<0.001$, one-way ANOVA followed by the Dunnett multiple comparison test, $\mathrm{n}=9$ /group. (E and F) Effects of repeated application of RTG on ipsilateral PWT (E) and PWL (F) in MRMT-1-inoculated rats. Note that repeated administration of RTG for 3 days produced long-term anti-allodynic and anti-hyperalgesic effects in bone cancer rats. ${ }^{* * *} \mathrm{P}<0.001$, two-way ANOVA followed by Bonferroni post-hoc test, $\mathrm{n}=9$ /group. $(\mathrm{G})$ Effects of repeated application of RTG on the locomotor function of rats measured by the angle of the inclined-plate at which the animal begins to fall. Note that repeated application of RTG had no significant damage on the locomotor function of rats ( $\mathrm{P}>0.05$, pre-drug vs. post-drug, two-tailed unpaired t-test, $\mathrm{n}=9 / \mathrm{group})$. RTG, retigabine; PWT, paw withdrawal threshold; PWL, paw withdrawal latency; ANOVA, analysis of variance; AUC, area under the time-course curve.

tumor-bearing mice exhibited sensitization to mechanical, heat, and cold stimuli that probably contributed to tumor-evoked hyperalgesia (29). In the present study it was suggested that although peripheral sensitization including the sensitization of primary sensory neurons participate in the generation of bone cancer pain $(1,8-10)$, central sensitization, especially the 
sensitization of dorsal horn WDR neurons, likely plays a more important role in tumor-evoked hyperalgesia and the maintenance of cancer-induced bone pain $(7,27,29,30,48)$.

To clarify the pathogenic mechanisms underlying the sensitization of dorsal horn WDR neurons in bone cancer rats, we focused on $\mathrm{KCNQ} / \mathrm{M}(\mathrm{Kv} 7)$ potassium channels, a family of voltage-gated potassium channels that is considered as a 'brake' to regulate the action potential firing and the neuronal excitability (16). Recently, we have found that inhibition of KCNQ/M channels in DRG neurons with XE-991 causes a robust increase in neuronal excitability that is associated with an obvious mechanical allodynia in naive rats (11). Consistent with our previous findings, the present study revealed that blockade of spinal KCNQ/M channels by local administration of XE-991, a potent $\mathrm{KCNQ} / \mathrm{M}$ channel blocker, induced a significant increase in C-fiber responses of dorsal horn WDR neurons in normal rats, implying that repression of spinal $\mathrm{KCNQ} / \mathrm{M}$ channels likely induces sensitization of dorsal horn WDR neurons that is highly related to the pathogenesis of pain hypersensitivity under cancer conditions $(48,49)$. In support of this notion, we discovered that intrathecal administration of XE-991 produced mechanical allodynia and thermal hyperalgesia in normal rats, suggesting that inhibition of spinal $\mathrm{KCNQ} / \mathrm{M}$ channels and its associated sensitization of dorsal horn WDR neurons probably contributed to the pathogenesis of pain hypersensitivity under cancer conditions. Moreover, we provide additional evidence showing that activation of spinal KCNQ/M channels by retigabine, a selective KCNQ/M channel opener, not only inhibited the bone cancer-induced hyperexcitability of dorsal horn WDR neurons, but also alleviated mechanical allodynia and thermal hyperalgesia in bone cancer rats, and all of these effects of retigabine were blocked by $\mathrm{KCNQ} / \mathrm{M}$-channel antagonist XE-991. These results are line with previous findings showing that in hemisected spinal cord from immature rats, blockade of $\mathrm{KCNQ} / \mathrm{M}$ channels with XE-991 induced depolarization and enhanced excitability of dorsal horn neurons, whereas activation of $\mathrm{KCNQ} / \mathrm{M}$ channels with retigabine produced hyperpolarization and a large decrease in the excitability of these neurons (35). Retigabine, applied to the dorsal spinal cord, inhibited $\mathrm{C}$ and A- $\delta$ fiber-mediated responses of dorsal horn neurons both in normal rats and in rats subjected to spinal nerve ligation (32).

In conclusion, the present study showed that inoculation of MRMT-1 tumor cells into the tibial canal in rats induced sensitization of dorsal horn WDR neurons in the spinal cord. Blockade of spinal KCNQ/M channels produced hyperexcitability of dorsal horn WDR neurons and pain hypersensitivity in normal rats, whereas activation of these channels inhibited the sensitization of dorsal horn WDR neurons, meanwhile alleviating mechanical allodynia and thermal hyperalgesia in bone cancer rats. Thus, suppression of KCNQ/M channels in the spinal cord leads to the sensitization of dorsal horn WDR neurons, thereby playing an important role in the development of cancer-induced bone pain.

\section{Acknowledgements}

The present study was supported by grants from the National Natural Science Foundation of China (81371237, 31171063 and 81072951), the Beijing Natural Science Foundation (7112079), the Special Foundation for Public Welfare Profession
Scientific Research Program from the Ministry of Health of the People's Republic of China (201302013-01) and the '973' Program of the Ministry of Science and Technology of China (2013CB531905).

\section{References}

1. Jimenez-Andrade JM, Mantyh WG, Bloom AP, Ferng AS, Geffre CP and Mantyh PW: Bone cancer pain. Ann NY Acad Sci 1198: 173-181, 2010.

2. Knopp KL, Nisenbaum ES and Arneric SP: Evolving cancer pain treatments: rational approaches to improve the quality of life for cancer patients. Curr Pharm Biotechnol 12: 1627-1643, 2011.

3. Middlemiss T, Laird BJ and Fallon MT: Mechanisms of cancer-induced bone pain. Clin Oncol (R Coll Radiol) 23: 387-392, 2011.

4. Paice JA and Ferrell B: The management of cancer pain. CA Cancer J Clin 61: 157-182, 2011.

5. von Gunten CF: Pathophysiology of pain in cancer. J Pediatr Hematol Oncol 33 (Suppl 1): S12-S18, 2011.

6. Luger NM, Mach DB, Sevcik MA and Mantyh PW: Bone cancer pain: from model to mechanism to therapy. J Pain Symptom Manage 29: S32-S46, 2005

7. Urch C: The pathophysiology of cancer-induced bone pain: current understanding. Palliat Med 18: 267-274, 2004.

8. Hamamoto DT, Khasabov SG, Cain DM and Simone DA: Tumor-evoked sensitization of $\mathrm{C}$ nociceptors: a role for endothelin. J Neurophysiol 100: 2300-2311, 2008.

9. Zhao J, Pan HL, Li TT, Zhang YQ, Wei JY and Zhao ZQ: The sensitization of peripheral C-fibers to lysophosphatidic acid in bone cancer pain. Life Sci 87: 120-125, 2010.

10. Zheng Q, Fang D, Cai J, Wan Y, Han JS and Xing GG: Enhanced excitability of small dorsal root ganglion neurons in rats with bone cancer pain. Mol Pain 8: 24, 2012.

11. Zheng Q, Fang D, Liu M, Cai J, Wan Y, Han JS and Xing GG: Suppression of KCNQ/M (Kv7) potassium channels in dorsal root ganglion neurons contributes to the development of bone cancer pain in a rat model. Pain 154: 434-448, 2013.

12. Wulff H, Castle NA and Pardo LA: Voltage-gated potassium channels as therapeutic targets. Nat Rev Drug Discov 8: 982-1001, 2009.

13. Biervert C, Schroeder BC, Kubisch C, Berkovic SF, Propping P, Jentsch TJ and Steinlein OK: A potassium channel mutation in neonatal human epilepsy. Science 279: 403-406, 1998.

14. Klinger F, Gould G, Boehm S and Shapiro MS: Distribution of M-channel subunits KCNQ2 and KCNQ3 in rat hippocampus. Neuroimage 58: 761-769, 2011.

15. Wang HS, Pan Z, Shi W, et al: KCNQ2 and KCNQ3 potassium channel subunits: molecular correlates of the M-channel. Science 282: 1890-1893, 1998.

16. Brown DA and Passmore GM: Neural KCNQ (Kv7) channels. Br J Pharmacol 156: 1185-1195, 2009.

17. Marrion NV: Control of M-current. Annu Rev Physiol 59: 483-504, 1997.

18. Shen W, Hamilton SE, Nathanson NM and Surmeier DJ: Cholinergic suppression of KCNQ channel currents enhances excitability of striatal medium spiny neurons. J Neurosci 25: 7449-7458, 2005

19. Fritch PC, McNaughton-Smith G, Amato GS, et al: Novel KCNQ2/Q3 agonists as potential therapeutics for epilepsy and neuropathic pain. J Med Chem 53: 887-896, 2010.

20. Gu N, Vervaeke K, Hu H and Storm JF: Kv7/KCNQ/M and $\mathrm{HCN} / \mathrm{h}$, but not $\mathrm{KCa} 2 / \mathrm{SK}$ channels, contribute to the somatic medium after-hyperpolarization and excitability control in CA1 hippocampal pyramidal cells. J Physiol 566: 689-715, 2005.

21. Hansen HH, Ebbesen C, Mathiesen C, et al: The KCNQ channel opener retigabine inhibits the activity of mesencephalic dopaminergic systems of the rat. J Pharmacol Exp Ther 318: 1006-1019, 2006.

22. Hansen HH, Andreasen JT, Weikop P, Mirza N, Scheel-Krüger J and Mikkelsen JD: The neuronal KCNQ channel opener retigabine inhibits locomotor activity and reduces forebrain excitatory responses to the psychostimulants cocaine, methylphenidate and phencyclidine. Eur J Pharmacol 570: 77-88, 2007.

23. Linley JE, Rose K, Patil M, Robertson B, Akopian AN and Gamper N: Inhibition of M current in sensory neurons by exogenous proteases: a signaling pathway mediating inflammatory nociception. J Neurosci 28: 11240-11249, 2008. 
24. Liu B, Linley JE, Du X, Zhang X, Ooi L, Zhang H and Gamper N: The acute nociceptive signals induced by bradykinin in rat sensory neurons are mediated by inhibition of M-type $\mathrm{K}^{+}$ channels and activation of $\mathrm{Ca}^{2+}$-activated Cl- channels. J Clin Invest 120: 1240-1252, 2010.

25. Rose K, Ooi L, Dalle C, Robertson B, Wood IC and Gamper N: Transcriptional repression of the $\mathrm{M}$ channel subunit Kv7.2 in chronic nerve injury. Pain 152: 742-754, 2011.

26. Woolf CJ: Central sensitization: implications for the diagnosis and treatment of pain. Pain 152: S2-S15, 2011.

27. Latremoliere A and Woolf CJ: Central sensitization: a generator of pain hypersensitivity by central neural plasticity. J Pain 10: 895-926, 2009

28. Donovan-Rodriguez T, Dickenson AH and Urch CE: Superficial dorsal horn neuronal responses and the emergence of behavioural hyperalgesia in a rat model of cancer-induced bone pain. Neurosci Lett 360: 29-32, 2004.

29. Khasabov SG, Hamamoto DT, Harding-Rose C and Simone DA Tumor-evoked hyperalgesia and sensitization of nociceptive dorsal horn neurons in a murine model of cancer pain. Brain Res 1180: 7-19, 2007.

30. Urch CE, Donovan-Rodriguez T and Dickenson AH: Alterations in dorsal horn neurones in a rat model of cancer-induced bone pain. Pain 106: 347-356, 2003.

31. Dedek K, Kunath B, Kananura C, Reuner U, Jentsch TJ and Steinlein OK: Myokymia and neonatal epilepsy caused by a mutation in the voltage sensor of the KCNQ2 $\mathrm{K}^{+}$channel. Proc Natl Acad Sci USA 98: 12272-12277, 2001.

32. Passmore GM, Selyanko AA, Mistry M, et al: KCNQ/M currents in sensory neurons: significance for pain therapy. J Neurosci 23: 7227-7236, 2003.

33. Passmore GM, Reilly JM, Thakur M, Keasberry VN, Marsh SJ, Dickenson $\mathrm{AH}$ and Brown DA: Functional significance of M-type potassium channels in nociceptive cutaneous sensory endings. Front Mol Neurosci 5: 63, 2012.

34. Rivera-Arconada I, Martinez-Gomez J and Lopez-Garcia JA: M-current modulators alter rat spinal nociceptive transmission: an electrophysiological study in vitro. Neuropharmacology 46 : 598-606, 2004

35. Rivera-Arconada I and Lopez-Garcia JA: Effects of M-current modulators on the excitability of immature rat spinal sensory and motor neurones. Eur J Neurosci 22: 3091-3098, 2005.
36. Rivera-Arconada I and Lopez-Garcia JA: Retigabine-induced population primary afferent hyperpolarisation in vitro. Neuropharmacology 51: 756-763, 2006

37. Roza C and Lopez-Garcia JA: Retigabine, the specific KCNQ channel opener, blocks ectopic discharges in axotomized sensory fibres. Pain 138: 537-545, 2008.

38. Zimmermann M: Ethical guidelines for investigations of experimental pain in conscious animals. Pain 16: 109-110, 1983

39. Medhurst SJ, Walker K, Bowes M, et al: A rat model of bone cancer pain. Pain 96: 129-140, 2002.

40. Liu M, Yang H, Fang D, et al: Upregulation of $\mathrm{P} 2 \mathrm{X} 3$ receptors by neuronal calcium sensor protein VILIP-1 in dorsal root ganglions contributes to the bone cancer pain in rats. Pain 154: 1551-1568, 2013.

41. Chaplan SR, Bach FW, Pogrel JW, Chung JM and Yaksh TL: Quantitative assessment of tactile allodynia in the rat paw. J Neurosci Methods 53: 55-63, 1994.

42. Dixon WJ: Efficient analysis of experimental observations. Annu Rev Pharmacol Toxicol 20: 441-462, 1980.

43. Zimmermann M: Pathobiology of neuropathic pain1. Eur J Pharmacol 429: 23-37, 2001.

44. Qu XX,Cai J,LiMJ,etal: Role of the spinal cord NR2B-containing NMDA receptors in the development of neuropathic pain. Exp Neurol 215: 298-307, 2009.

45. Rivlin AS and Tator $\mathrm{CH}$ : Objective clinical assessment of motor function after experimental spinal cord injury in the rat. J Neurosurg 47: 577-581, 1977.

46. Xing GG, Liu FY, Qu XX, Han JS and Wan Y: Long-term synaptic plasticity in the spinal dorsal horn and its modulation by electroacupuncture in rats with neuropathic pain. Exp Neurol 208: 323-332, 2007.

47. Lu Y and Perl ER: Selective action of noradrenaline and serotonin on neurones of the spinal superficial dorsal horn in the rat. J Physiol 582: 127-136, 2007.

48. Yanagisawa Y, Furue H, Kawamata T, et al: Bone cancer induces a unique central sensitization through synaptic changes in a wide area of the spinal cord. Mol Pain 6: 38, 2010.

49. Gordon-Williams RM and Dickenson AH: Central neuronal mechanisms in cancer-induced bone pain. Curr Opin Support Palliat Care 1: 6-10, 2007. 